\title{
TOMMI - Deutscher Kindersoftwarepreis 2019: Die Sieger stehen fest
}

http://doi.org/10.1515/bd-2020-0005

Sie kamen, spielten und prämierten: 3.331 Kinder durften wochenlang in rund 20 Bibliotheken knapp 40 Spiele testen, und dabei das Innere von Bienenwaben erforschen, Monster fangen, Roboter und Murmelbahnen bauen. Jetzt stehen endlich die Sieger im Medienkompetenz-Projekt TOMMI fest. Auf der Frankfurter Buchmesse übergaben Kinder am 18.10.2019 die Auszeichnungen an die Gewinner. In der Kategorie Konsole räumte „Yoshi’s Crafted World“ (Nintendo) und bei PC „Rug Tales“ (Purple Hills) ab. Zu den weiteren Gewinnern gehören in der Sparte Apps „Thinkrolls Space“ (Avokiddo). Sieger im Genre Elektronisches Spielzeug wurde „Sphero“ (Sphero).

„Wir sind sehr glücklich, dass so viele Kinder Jahr für Jahr die Gelegenheit ergreifen, bei diesem bundesweiten Partizipationsprojekt in Bibliotheken engagiert mitzuwirken“, erklärt Co-Initiator Marko Petersen, Geschäftsführer der Family Media in Freiburg. „Unser Dank gebührt den Öffentlichen Bibliotheken, die mit ihrem geschulten Personal die Tests mit großem Aufwand durchführten“, meint Co-Initiator Thomas Feibel von FEIBEL.DE, Büro für Kindermedien in Berlin.

Der Deutsche Kindersoftwarepreis TOMMI steht unter der Schirmherrschaft der Bundesfamilienministerin Dr. Franziska Giffey und wird von der Family Media (Freiburg) und FEIBEL.DE - Büro für Kindermedien (Berlin) herausgegeben. Partner des Preises sind der Deutsche Bibliotheksverband e.V. (dbv), die Frankfurter Buchmesse, Google, jugendschutz.net, Partner \& Söhne und das ZDF.

\section{Sonderpreis Kindergarten \& Vorschule}

Den Sonderpreis Kindergarten \& Vorschule erhielt das Spiel „Fiete World“. Prof. Dr. Stefan Aufenanger von der Johannes-Gutenberg-Universität in Mainz führte mit seinem Team die Tests der fünf Spielangebote in Kindergärten durch. 


\section{Die Fachjury 2019}

Vorsitz: Thomas Feibel (familie\&co, FEIBEL.DE).

Prof. Dr. Stefan Aufenanger (Universität Mainz), Prof. Dr. Linda Breitlauch (Hochschule Trier), Christoph Drösser (Freier Journalist in San Francisco), Martin Eisenlauer (Bild am Sonntag), Julia Fastner (jugendschutz.net), Stephan Freundorfer (Journalist), Tim Gailus (KIKA, „Timster“), Fabienne Zähringer (ZDF-Kinder- und Jugendprogramm), Carsten Görig (Spiegel Online), Moses Grohé (love4games.org), Steffen Haubner (Kölner Stadtanzeiger), Andreas Klinger (Stadtbücherei Biberach), Karol Kleinert (Schüler, 12 Jahre), Bertram Küster (Bild.de), Anatol Locker (Journalist), Dr. Kathrin Mertes (Universität Mainz), Kurt Sagatz (Tagesspiegel), Linda Scholz (Spieleratgeber-NRW), Prof. Dr. Friederike Siller (Fachhochschule Köln), Jürgen Sleegers (Fachhochschule Köln), Dr. Michael Spehr (Frankfurter Allgemeine Zeitung).

\section{In diesen Bibliotheken wurden die Spiele getestet:}

Baden-Württemberg

Bayern

Berlin

Brandenburg

Hamburg

Hessen

Mecklenburg-Vorpommern

Niedersachsen

Nordrhein-Westfalen

Sachsen

Sachsen-Anhalt

Schleswig-Holstein

Neu: Österreich

\author{
Stadtbücherei Biberach an der Riß - Medien- \\ und Informationszentrum \\ Stadtbibliothek Ludwigsburg \\ Stadtbibliothek Freiburg \\ Stadtbibliothek Pforzheim \\ Münchner Stadtbibliothek am Gasteig \\ Stadtbibliothek Unterschleißheim \\ Stadtbibliothek Berlin-Lichtenberg: Anna- \\ Seghers-Bibliothek \\ Gemeindebibliothek Blankenfelde-Mahlow \\ Bücherhallen Hamburg: Bücherhalle Alstertal \\ Stadtbücherei Frankfurt am Main: Bibliotheks- \\ zentrum Sachsenhausen \\ Stadtbibliothek Ribnitz-Damgarten \\ Stadtbibliothek Hannover \\ Stadtbibliothek Wolfsburg \\ Stadtbibliothek Leverkusen \\ Stadtbibliothek Euskirchen \\ Leipziger Städtische Bibliotheken \\ Stadt- und Schulbibliothek Landsberg \\ Stadtbücherei Kiel \\ Büchereien Wien
}




\section{Gut zu wissen - die Ziele des TOMMI:}

- Der TOMMI macht gute Apps, Computer- und Konsolenspiele sowie elektronisches Spielzeug einem größeren Publikum bekannt und verschafft den Eltern damit einen Überblick im Spiele-Dschungel.

- Der TOMMI setzt sich positiv mit dem Thema Computer- und Konsolenspiele auseinander.

- $\quad$ Der TOMMI bindet über 3.000 Kinder in die Jurytätigkeit ein, erzieht so zum kritischen Umgang mit Computer- und Konsolenspielen und fördert nachhaltig die Medienkompetenz.

- Der TOMMI präsentiert qualitativ hochwertige Spiele und schützt vor Spielinhalten, die für Kinder nicht geeignet sind.

- Der TOMMI hilft Eltern, ihre Kinder im Medienzeitalter zu fördern und zu begleiten.

\section{Die Sieger (Kurzfassung)}

\section{Konsole}

1 Switch: Yohi's crafted world

Nintendo

2 Switch: Super Mario Maker 2

Nintendo

3 Switch: Pokémon Lets go Pikachu!; Lets go, evoli

Nintendo

\section{Apps}

1 Thinkrolls Space

Avokiddo

2 Fiete World

Ahoii

3 Shine - Reise des Lichts

Fox \& Sheep

\section{PC}

1 Rug Tales

2 Landwirtschafts-Simulator 2019

Purple Hills

3 Unrailed!

Astragon

Daedalic 


\section{Elektronisches Spielzeug}

1 Sphero Bolt

2 GraviTrax mit neuen Actionsteinen

3 Tori
Sphero

Ravensburger

Bandai Namco

\section{Kindergarten und Vorschulpreis}

1 Fiete World

Ahoiii

\section{Begründungen}

\section{Konsole}

\section{Platz 1: Yoshi's Crafted World (Nintendo)}

Das sagt die Fachjury: Yoshi ist einfach süß. Er fängt Gegner mit seiner langen Zunge, schluckt sie runter und scheidet sie dann als Ei wieder aus. Mit dem schießt er wiederum Gegner aus dem Weg oder ballert Teile der Kulissen weg, um versteckte Münzen oder Wege zu finden. Wunderbar sind die Kulissen, die allesamt aussehen, als ob sie am Basteltisch im Kinderzimmer entstanden wären. Toll auch, dass es bei dem Jump'n“ Run zwei verschiedene Schwierigkeitsstufen gibt, sodass auch Ungeübte wenig Frust spüren und gerne weiterspielen.

\section{Ab 6 Jahren.}

Das sagt die TOMMI-Kinderjury: „Yoshi‘s Crafted World“ soll beim TOMMI Platz 1 gewinnen, weil es das voll verdient. Es ist superbunt, superlustig und vor allem megasüß. Uns gefällt die sehr fröhliche Stimmung und die tolle Grafik, die einfach gute Laune macht. Wir können zu zweit spielen, Charaktere aussuchen und auch selbst kreativ werden. Die Aufgaben und Rätsel sind toll und wir müssen auch strategisch denken. Es gab immer eine gute Erklärung, man kann jederzeit nachschauen. Wir empfinden nur Positives im Spiel. 


\section{Platz 2: Super Mario Maker 2 (Nintendo)}

Das sagt die Fachjury: Mit „Super Mario Maker 2“ kann jeder recht einfach Jump’n“ Run Level im „Super Mario“-Stil bauen. Mit den Fingern verschieben Kinder auf dem Touchpad der Switch die Kulissen, setzen Gegner in die Levels oder verteilen Geräusche, die loströten, wenn sie eine bestimmte Stelle erreichen. Das macht viel Spaß, fordert aber auch heraus. Schön, dass die fertigen Werke mit anderen Spielern geteilt oder deren Kreationen getestet und umgebaut werden können. Wer nicht nur bauen will, kann auch erstmal spielen - natürlich ein Spiel im „Super Mario“-Stil.

Ab 8 Jahren.

Das sagt die TOMMI-Kinderjury: „Super Mario Maker 2“ soll beim TOMMI Platz 2 gewinnen, weil wir so leicht unsere eigenen Game-Designer werden können. Juhuuu! Es macht wirklich sehr viel Spaß, dank umfangreicher Werkzeuge unsere eigenen Mario-Levels zu bauen und der Kreativität freien Lauf zu lassen. Andere dürfen sich dann daran die Zähne ausbeißen. Auch online. Die Levels des Abenteuermodus machen Spaß. Bowser besiegen ist uns immer wieder eine Freude. An diesem Spiel ist alles pikobello.

\section{Platz 3: Pokémon Let’s Go Pikachu; Let's Go Evoli (Nintendo)}

Das sagt die Fachjury: Wird ein normales Pokémon-Spiel mit Mechaniken aus „Pokémon Go“ verbunden, entsteht so etwas wie „Pokémon: Let’s Go, Pikachu!“ Die Faszination mit den kleinen Monstern, das Sammeln und Kämpfen, alles ist hervorragend umgesetzt und macht auch Anfängern im Pokémon-Universum Spaß. Wenn Spieler dann noch mit einer schnellen Bewegung des Switch-Controllers einen Poke-Ball werfen, macht das richtig Vergnügen. Für jüngere Spieler gibt es noch die Möglichkeit, mit einer weiteren Trainer-Figur auf dem Bildschirm herumzulaufen.

Ab 8 Jahren.

Das sagt die TOMMI-Kinderjury: „Pokémon Let’s Go Pikachu; Let’s Go Evoli” soll beim TOMMI den 3. Platz gewinnen, weil es ein Kultspiel ist. Es ist zwar ein neues Spiel, aber zum Glück seiner Grundidee treu geblieben: Pokémon fangen und trainieren. Manche lassen sich allerdings zu leicht fangen. Aber die Pokémons sind wirklich sooo süß. Die Kämpfe sind immer wieder richtig spannend. Auch junge Kinder haben eine gute Chance, weil wirklich alles gut erklärt und gut verständlich ist. Das Spiel ist richtig cool. 


\section{Apps}

\section{Platz 1: Thinkrolls Space (Avokiddo)}

Das sagt die Fachjury: Das farbenfrohe Touchscreen-Puzzle schickt Kinder mit einem rollenden Alien auf zahllose Planeten, wo er einen Schlüssel berühren und zum Ausgang gelangen muss. Milde bis ordentlich herausfordernd, aber nie frustrierend, geht es durch mehr als 200 Level. Verschwindende Brücken, diverse Objekte, Teleporter, Gruben oder Käseflöze sorgen für Abwechslung und fordern vorausschauendes Denken. „Thinkrolls Space“ ist eine durchdachte Puzzle-App, die ohne nervige Free-to-Play-Mechanismen zum Grübeln und Grinsen anregt.

Ab 8 Jahren.

Das sagt die TOMMI-Kinderjury: „Thinkrolls Space“ soll beim TOMMI den 1. Platz gewinnen, weil wir uns richtig anstrengen und viel nachdenken müssen, um die Hindernisse zu überwinden. Denn das Denk- und Rätselspiel ist nicht einfach, aber auch nicht zu schwer. Es macht Spaß, verschiedene Wege zu suchen. Nirgends gibt es so viele witzige Aliens und Monster. Das Käsemonster oder das Kuchenfüttern bringt uns richtig zum Lachen. Das Spiel ist total fantasievoll und für jede Altersstufe geeignet. Man kann es auch zu zweit spielen.

\section{Platz 2: Fiete World (Ahoiii)}

Das sagt die Fachjury: Fiete entführt sehr junge Kinder in ein kunterbuntes, seitlich scrollendes Wimmelbuch, in dem es unzählige witzige Details zu entdecken gibt. Da lässt sich endlos Unfug treiben - ein Pferd wird zum Traktorfahrer gemacht oder ein halber Hausstand im Plumpsklo versenkt. Aber auch ein Blumenbeet darf bestellt oder ein Schokoladenkuchen gebacken werden. Und das alles in Fietes ländlicher Heimat am Meer. Drei weitere Szenarien - Schatzinsel, Frankreich, Indien - lassen sich bei Gefallen durch In-App-Käufe freischalten.

Ab 4 Jahren.

Tipp: Um unerwünschte Käufe zu verhindern, lassen sich In-App-Käufe in den Einstellungen deaktivieren bzw. durch ein Passwort schützen.

Das sagt die TOMMI-Kinderjury: „Fiete World“ soll beim TOMMI den 2. Platz gewinnen, obwohl wir eigentlich viel zu alt dafür sind. Aber es ist sehr faszinierend, ein Open World-Spiel zu spielen. Am Anfang ist diese große Freiheit ungewohnt, weil es keine Geschichte gibt. Aber es ist einfach alles möglich: Traktor fahren, kochen, Zähne putzen und Würstchen grillen. Es gibt Waffen, aber man kann niemanden töten. Alles lässt sich herumschieben und regt zum Experimentieren an. Auch toll: Wir müssen zum Glück nichts lesen. 


\section{Platz 3: Shine (Fox \& Sheep)}

Das sagt die Fachjury: Diese App ist sehr schön gestaltet: Die Atmosphäre, die Musik und die grafische Darstellung fallen sofort als große Stärke auf. Als kleine Lichtkugeln müssen die anderen Moonies in den verschiedenen Leveln eingesammelt werden. Die Steuerung funktioniert intuitiv über einfache Tipp-Gesten. Schafft man es nicht rechtzeitig zur nächsten Lichtkugel, geht das eigene Licht aus. Die Kinder können jedoch direkt wieder neu starten. Mit vier Kapiteln à zehn Leveln ist „Shine“ angenehm umfangreich, sammelbare dunkle Kugeln sorgen für einen hohen Wiederspielwert.

Ab 6 Jahren.

Das sagt die TOMMI-Kinderjury: „Shine“ soll beim TOMMI den 3. Platz gewinnen, weil es im Gegensatz zu vielen anderen Spielen überhaupt nicht hektisch ist. Wir sind das Licht, müssen dafür sorgen, dass es nicht ausgeht und andere Lichter einsammeln. Es hat zwar eine sehr einfache Mechanik, aber die Stimmung, die tolle Grafik und die schöne Musik und die guten Soundeffekte sind sehr entspannend und beruhigend. Mit Kopfhörer ist es noch besser. Mit „Shine“ kann man nach einem anstrengenden Schultag richtig gut runterkommen.

\section{PC}

\section{Platz 1: Rug Tales (Purple Hills)}

Das sagt die Fachjury: Das nette Setting auf dem Teppich (Rug) lässt einen immer wieder schmunzeln. Zum Beispiel, wenn du mit dem Spielzeugauto über die Mauer aus Bauklötzen springst. Fäden und Büroklammern sind die Rohstoffe, um Brücken und Gebäude zu bauen. Die Geschichte ist kindgerecht, aber nicht langweilig, die Figuren sehr sympathisch. $\mathrm{Zu}$ Beginn nimmt einen das Spiel gut an die Hand, sodass Kinder immer wissen, was sie zu tun haben. Später werden die Aufgaben komplexer, aber nie zu schwierig. Zur Not ist sogar eine Komplettlösung dabei.

\section{Ab 8 Jahren.}

Das sagt die TOMMI-Kinderjury: „Rug Tales“ soll beim TOMMI den 1. Platz gewinnen, weil wir sofort von dieser fantasievollen Teppichwelt gefesselt sind. In diesem Teppichland lebt ein Bauer, der hat sich in eine Frau verliebt. Die wird eines Tages weggefegt und der Bauer macht sich auf, sie zu retten. Das Spiel ist trotzdem fröhlich, aber nicht dramatisch. Die Grafik ist bunt und scharf und man kann jeden Grashalm erkennen. Es gefällt uns sehr, dass es sehr viele Rätsel gibt, die herausfordernd, aber nie zu schwierig sind. 


\section{Platz 2: Landwirtschafts-Simulator 2019 (Astragon)}

Das sagt die Fachjury: Der „Landwirtschafts-Simulator“ führt den Spieler in die Grundlagen der Agrarwirtschaft ein. Auf Karten erkunden Kinder Landschaften und bewirtschaften mit landwirtschaftlichen Maschinen eigenen Grund und Boden. Dazu gehört auch das Säen und Ernten von Nutzpflanzen und die Haltung und Versorgung von Nutztieren. Darüber hinaus lernen Kinder die alltägliche Praxis und die wirtschaftlichen Herausforderungen der Landwirtschaft bestens kennen. Das Spiel ist also für Kinder, die großes Interesse an der Landwirtschaft haben, perfekt.

Ab 10 Jahren.

Das sagt die TOMMI-Kinderjury: Der „Landwirtschafts-Simulator 2019“ soll den 2. Platz beim TOMMI gewinnen, weil wir uns als richtige Farmer fühlen und hocharbeiten können. Wir dürfen Traktor fahren, pflügen, aussäen, ernten, Tiere versorgen, Bäume fällen und Geld verdienen. Das Spiel ist nicht leicht, weil es so viele Möglichkeiten bietet, aber Popup-Nachrichten erklären alles sehr gut. Dadurch wissen wir, was zu tun ist und wir können dabei sogar etwas lernen. Der Nachtmodus ist super. Das Spiel ist ein tolles Simulationsspiel.

\section{Platz 3: Unrailed! (Daedalic)}

Das sagt die Fachjury: In „Unrailed!“ müssen Gleise vervollständigt werden, damit der eigene Zug sicher bis zur nächsten Station fahren kann. Die Steuerung funktioniert sehr einfach und lässt sich gut bedienen. Die niedliche KlötzchenOptik ist bunt und kreativ. Die Spielmechanik und die Idee sind sehr innovativ. Besonders viel Spaß macht es, mit Freunden gemeinsam zu versuchen, die Gleise für den Zug zu ebnen. Das Spiel erfordert Zeitmanagement, taktisches Denken, strategisches Planen und gemeinsame Absprachen.

Ab 8 Jahren.

Das sagt die TOMMI-Kinderjury: „Unrailed!“ soll beim TOMMI den 3. Platz gewinnen, weil es ein supercooles Eisenbahnspiel ist. Wir müssen eine Bahnstrecke bauen, die den nächsten Bahnhof erreicht und wir sollen uns dabei sehr beeilen, weil sonst der Zug kaputt geht. Grafik und Design gefällt uns besonders gut, weil sie uns an „Minecraft“ erinnert. Die Steuerung ist gut und einfach zu verstehen. Wir hätten gerne eine Kuh in den Transporter gestellt, aber Diebe vertreiben ist auch okay. Es ist ein Spiel, das man sehr gut zu zweit spielen kann. 


\section{Elektronisches Spielzeug}

\section{Platz 1: Sphero Bolt (Sphero)}

Das sagt die Fachjury: „Sphero Bolt“ ist ein kugelförmiger Roboter, der über eine App gesteuert wird. Das Modell unterscheidet sich von seinen Vorgängern durch eine programmierbare LED-Matrix. Vollgepackt mit Infrarotsensoren, einem Lichtsensor, einem Kompass, einem Gyroskop, einem Beschleunigungsmesser sowie Motorgeber rollt er so, wie noch nie ein Ball zuvor gerollt ist. „Sphero Bolt“ kann Kindern die Grundlagen des Programmierens näherbringen, für Bildungszwecke wird jedoch die Einbettung in ein pädagogisches Konzept empfohlen.

Ab 8 Jahren.

Das sagt die TOMMI-Kinderjury: „Sphero Bolt“ soll beim TOMMI den 1. Platz gewinnen, weil diese durchsichtige Kugel ein richtiger Roboter ist. Sie mit dem iPad zu lenken macht besonders viel Spaß. Wir geben den Weg ein und „Sphero“ bewegt sich dahin. Wir haben viele verschiedene Parcours gebaut. Die Kugel kann leuchten und sprechen und ist für Fortgeschrittene ein Programmierspaß. Wir denken, dass die Kugel für Kinder ab 0 Jahren geeignet ist, wenn die Eltern sie steuern und die Kinder hinterherkrabbeln.

\section{Platz 2: Kugelbahn mit App: GraviTrax mit neuen Actionsteinen (Ravensburger)}

Das sagt die Fachjury: „GraviTrax“ bereitet von Anfang an Freude. Mit verschiedenen Bauelementen lassen sich unterschiedlichste Bahnen bauen. Das Spiel beinhaltet sehr viele Komponenten, sodass es unendlich viel zu entdecken gibt. Dreidimensionales und logisches Denken werden gefordert. Tolles Spiel für jede Altersklasse! Die dazugehörige App bietet zusätzlich digitale Unterstützung und Anleitungen beim Bahnenbau. So können selbst Kinder, die noch nicht lesen können, Spaß mit „GraviTrax“ haben. Digitales und Haptik gehen hier eine besondere Kombination ein.

Ab 6 Jahren.

Das sagt die TOMMI-Kinderjury: „GraviTrax“ soll beim TOMMI den 2. Platz gewinnen, weil es App und Spielzeug auf wunderbare Weise verbindet. So eine Kugelbahn bietet endlose Möglichkeiten, und wir lernen viel über Schwerkraft. Es ist ein richtiges Bauprojekt und wird nie langweilig, weil jede Bahn anders ist. Erst wird nach Anleitung mit der App gebaut und wenn die Kugel rollt, halten wir vor Spannung den Atem an. Es gibt so viele Bauteile, wie zum Beispiel Brücken oder das Trampolin. Es ist toll, dass wir die Kugel zum Schleudern, Hüpfen und Fliegen bringen. Schon alleine darum kommen wir zurück in die Bibliothek. 


\section{Platz 3: Tori (Namco Bandai)}

Das sagt die Fachjury: Bei „Tori“ verschwimmen Realität und Fiktion wie bei kaum einem anderen Spiel. Hinzu kommt, dass die Kreativität des Nutzers stark gefordert wird: Kinder können selbst gestaltete Gegenstände in das Spiel integrieren. So werden individuelle und einzigartige Spielwelten erschaffen. Während „Tori Supreme Builder“ zum einen das räumliche Denken fordert, lösen Kinder bei „Tori Jungle Rescue“ verschiedene Rätsel. Durch die hohe Interaktivität und die 3D-Optik entsteht ein hoher Fun-Faktor. Sehr fesselnd.

Vorsicht aus Jugendschutz-Sicht: Die App überträgt Daten, wie z. B. die NutzerID und den Gerätenamen, an externe Server. Das könnte zur Profilbildung benutzt werden. Tipp: Die App offline nutzen!

Ab 6 Jahren.

Das sagt die TOMMI-Kinderjury: „Tori“ soll beim TOMMI den 3. Platz gewinnen, weil es eine Kreativkiste ist und Malen, Basteln und Spielspaß zusammenbringt. Erbauen wir ein cooles Raumschiff zusammen und malen es an, dann kann es auf der App sogar richtig fliegen. Dabei müssen wir richtig gut steuern, weil es viele Hindernisse gibt. Wenn wir mit einem Katapult Dinge abschießen, dann ist richtig Action angesagt. Die Level sind sehr abwechslungsreich. „Tori“ ist selbsterklärend und die Grafik war echt Bombe.

\section{Sonderpreis Kindergarten \& Vorschule}

\section{Fiete World (Ahoiii)}

Das sagen die Medienpädagogen: Mit weitem Abstand vor all den anderen Apps und Spielen hat „Fiete World“ das Herz der Kinder erobert. Dort gibt es tolle Gelegenheiten, sich spielerisch auszuprobieren, in andere Figuren hineinzuversetzen und eine eigene kleine Welt zu bauen. Mit der Möglichkeit, alles mit einem Knopfdruck noch als Video aufzunehmen, werden schon die Jüngsten kleine Filmmacher und ihre Medienkompetenz wird gefördert. Durch das freie Erkunden in der Open World von Fiete können Kinder sich selbst erproben, in verschiedene Rollen schlüpfen und damit mit Spaß ihrer Kreativität und Fantasie freien Lauf lassen. Genau das machen medienpädagogisch wertvolle Anwendungen für Kinder aus. 


\section{Pressekontakte:}

\section{Feibel.de, Büro für Kindermedien}

Thomas Feibel

Jenaerstr. 15, 10717 Berlin

Tel.: 030 85733030, E-Mail: tom@feibel.de

URL: http://www.kindersoftwarepreis.de, http://www.feibel.de

\section{Deutscher Bibliotheksverband e.V. (dbv)}

Kristin Bäßler, Leitung Kommunikation / Pressesprecherin

Tel.: 030 / 6449899 25, E-Mail: presse@bibliotheksverband.de

URL: www.bibliotheksverband.de 